\title{
Skill training, retention, and transfer: The effects of a concurrent secondary task
}

\author{
ALICE F. HEALY, ERICA L. WOHLDMANN, JAMES T. PARKER, and LYLE E. BOURNE, JR. \\ University of Colorado, Boulder, Colorado
}

\begin{abstract}
In two experiments, we examined training, retention, and transfer of a duration production skill in a prospective paradigm. Participants were trained with feedback and then were either tested immediately for transfer without feedback or retrained with feedback 1 week later. There were three training and retraining conditions, two involving secondary tasks. Retention of the duration production skill was perfect across the 1-week delay when the secondary task condition was unchanged, but there was no skill transfer when that condition was changed. These findings demonstrate specificity of training, with the assumption that the cognitive operations learned during duration production training incorporate requirements of the secondary task. More generally, this study challenges the current practice in which training conditions often do not match eventual testing conditions.
\end{abstract}

Research on skill acquisition and retention has demonstrated that performance of a learned skill on a transfer test tends to be specific to the actual items practiced and the context in which they are practiced (e.g., Rickard, Healy, \& Bourne, 1994). An issue addressed in the present study is whether performance of such a skill is also specific at transfer to changes in a concurrent secondary task. More generally, the aim of the present study is to examine the effects of changes in a concurrent secondary task on training, retention, and transfer of a skill.

Skill specificity has been demonstrated in a variety of tasks. For example, Clawson, King, Healy, and Ericsson (1995) found evidence for lasting specificity of training effects in the Stroop task. Participants who were trained to name colors and ignore words were faster after training on the specific trained color set, relative to an untrained set. Likewise, Rickard et al. (1994) used a mental calculation task to train participants on single-digit multiplication and division problems. They found that the effects of training in this mental calculation task were highly specific to the trained problems. Participants were faster on problems actually practiced than on totally new problems and faster on practiced problems than on the same problems altered

This research was supported in part by Army Research Institute Contracts DASW01-96-K-0010, DASW01-99-K-0002, and DASW01-03$\mathrm{K}-0002$ to the University of Colorado. Experiment 1 was included in J.T.P.'s master's thesis and was reported at the 41 st Annual Meeting of the Psychonomic Society, 2000, in New Orleans (Parker, Healy, \& Bourne, 2000). We are grateful to Lewis Harvey, a member of the master's thesis committee, for helpful suggestions concerning this research, to Andrew Hsiao and Peter Killeen for helpful comments on earlier versions of this article, and to Anne Catalano for help with the analysis of the secondary task in Experiment 2. Correspondence should be addressed to A. F. Healy, Department of Psychology, University of Colorado, 345 UCB, Boulder, CO 80309-0345 (e-mail: ahealy@psych.colorado.edu). by either changing the operations (e.g., multiplication to division) or changing the operand order.

The task investigated in the present study is duration production, in which individuals produce time intervals of various given lengths. The time interval to be produced is filled with a concurrent secondary task, so that the examination of the effects of changes in the secondary task on performance of the duration production primary task can be assessed. Because duration estimation has been shown to be greatly affected by what occurs during the interval (see, e.g., Hicks, Miller, \& Kinsbourne, 1976, and more recently, Sawyer, 1999, for reviews), the presence of a secondary task would be expected to have robust effects on the duration production skill.

Specificity in transfer tasks can be interpreted in terms of several related theoretical proposals. By the principle of procedural reinstatement, durable retention of a learned skill is best produced by duplicating the exact learning procedures during testing (Healy et al., 1992), so that a durable skill is expected to lack generalizability (Healy \& Bourne, 1995). Similarly, Kolers and Roediger (1984) have argued that memory is crucially dependent on mental procedures acquired during learning. Furthermore, the principles of both transfer-appropriate processing (e.g., Morris, Bransford, \& Franks, 1977) and encoding specificity (e.g., Tulving \& Thomson, 1973) emphasize the match between encoding and retrieval operations. All of these various views can be seen as derived from Thorndike's (1906) theory of identical elements of transfer, according to which transfer occurs to the extent that elements overlap. Singley and Anderson's (1989) principle of "use specificity of procedural knowledge" was also based on Thorndike's theory and is related conceptually to these other accounts, with the elements in this case viewed as production rules. Rickard and Bourne's (1995) identical-elements model of arithmetic skills was also motivated by Thorndike's account, but the elements in their model were abstract representations of numbers 
and arithmetic operations, rather than the stimulus items themselves.

In apparent contradiction to predictions based on the hypothesis that a learned skill will be specific to the trained items, Fetterman and Killeen (1990) found that a counting strategy in duration reproduction can be maintained fairly constantly across a range of time intervals. This finding suggests that a counting strategy used in producing durations might be highly generalizable across at least some task dimensions. Because of this challenging finding, the present study provides a critical test of the specificity of training for the skill of duration production.

To date, specificity of training has been shown only in situations involving a single task. The question arises, for a dual-task situation, of whether a change in a secondary task would affect performance on the primary task. Thus, in the present study, we incorporated various secondary task requirements that were to be performed concurrently with duration production, and we examined whether changes in the secondary task requirements during transfer testing would disrupt duration production performance. Such a disruption would be expected if specificity of training applies to secondary tasks, as well as to primary tasks. This outcome might occur, for example, if the primary and the secondary tasks were to merge into a single functional task from the perspective of the participant. This idea is considered at greater length in the General Discussion section.

\section{Contextual Interference}

The principle of contextual interference asserts that high interference during training should slow learning but often produce better retention and greater transfer even when training time is equated (e.g., Battig, 1972, 1979). Thus, retention of an acquired skill might be promoted by learning conditions with interfering information. One method for creating contextual interference (e.g., Schneider, Healy, Ericsson, \& Bourne, 1995; Shea \& Morgan, 1979) employs blocked and mixed practice schedules during skill acquisition. Blocked schedules consist of sequences of trials all of the same type, whereas mixed schedules consist of sequences of trials differing in type. By a contextual interference account, duration production would improve more during training in a blocked practice schedule than in a mixed practice schedule, but retention and transfer would be worse for the blocked schedule than for the mixed schedule. On the basis of finding that several different manipulations of task difficulty yielded results similar to those produced by the practice schedule manipulation, Schneider, Healy, and Bourne (2002) suggested that any manipulation of task difficulty during training may have facilitating effects during retention and transfer testing. According to this difficulty-of-training hypothesis, a more difficult dual task during training might yield superior duration production accuracy during transfer testing.

\section{Present Study}

The aim of the present study was to examine the effects of various training conditions on retention and transfer of the skill of short interval (less than $1 \mathrm{~min}$ ) duration production. Blocked and mixed practice schedules were included in order to evaluate contextual interference effects on training, retention, and transfer. On a given trial during training, participants produced a specified duration based on a fixed arbitrary unit of time. They were given immediate feedback as to the accuracy of their response. In Experiment 1, this training phase was immediately followed by a testing (i.e., transfer) phase without feedback. In Experiment 2, the training phase occurred 1 week before a retraining phase, both of which included feedback.

In most of the previous studies, clock time (normal fixed units of seconds or minutes) was used as a basis of duration judgments (e.g., Zakay, 1990). Because participants have prior everyday experience with the estimating of seconds or minutes, we chose a slightly different unit of time in order to highlight the effects of training on the skill of duration production. In our paradigm, a fixed arbitrary unit of time was chosen, with 1 unit equal to 47 computer "ticks," or approximately 47/60ths of a second $(783 \mathrm{msec})$. The participants were not told the duration of a unit; they learned this information through feedback provided on each trial, so they were forced to base their productions purely on guessing at the start of training.

\section{EXPERIMENT 1}

In a prospective production paradigm, participants were trained across blocks of trials with different intervals of time, expressed in the fixed arbitrary units. In the blocked training condition, all of the trials in a given block were for the same interval, whereas in the mixed training condition, each trial in a block was for a different interval. Immediately after training, the participants were given a transfer test that included old, actually practiced intervals intermixed with new intervals both within and outside the practice range. If training is specific to the trained intervals, performance at transfer should be better for the old actual intervals than for new intervals and should be better for new intervals within the practice range than for those outside the practice range. Alternatively, on the basis of the work of Fetterman and Killeen (1990), at transfer there should be no difference among the various types of intervals.

We also expected that production accuracy during training would improve across blocks of trials and, on the basis of contextual interference (e.g., Battig, 1972, 1979; Schneider et al., 2002; Schneider et al., 1995), would be better for the blocked than for the mixed practice schedule. On the basis of the same principle, during transfer (which always followed a mixed practice schedule), production accuracy might be better for those participants trained with a mixed practice schedule than for those trained with a blocked practice schedule.

We compared three training conditions, all of which required the production of fixed arbitrary intervals of time, but two of which were intended to suppress counting. Specifically, we compared a control condition, without a 
secondary task, with two conditions with secondary tasks, one relatively easy and the other more difficult, but both involving articulation. It is generally accepted that duration judgments are deliberate and based on explicit strategies, among which rhythmic counting is the preferred strategy (e.g., Killeen \& Weiss, 1987). Because a counting strategy usually requires subvocalization, it should be inhibited in the difficult condition and perhaps even in the easy condition, given the articulation demands of the secondary task (see, e.g., Healy \& Nairne, 1985). Thus, it is predicted that duration production during training should be best in the control condition and worst in the difficult secondary task condition. Testing always occurred with no secondary task. If training is specific to the secondary task, transfer performance should be worse for the easy and difficult conditions than for the control condition. A conflicting prediction would be made on the basis of the training difficulty hypothesis, which is the extended version of the contextual interference hypothesis raised by Schneider et al. (2002). According to these ideas, training under the difficult condition should yield superior duration production accuracy during transfer, relative to training under either the easy or the control condition.

\section{Method}

\section{Design}

The primary task involved learning to produce fixed intervals of time expressed in arbitrary units, with 1 unit (unbeknownst to participants) equal to $783 \mathrm{msec}$. Each participant was trained with feedback for six blocks of 6 trials and then was tested immediately in transfer for two blocks of 12 trials without feedback. For the transfer trials, we included four training intervals (old actual) and four new intervals of each of two types (new within the practice range, new outside the practice range).

We included three different training conditions. In the control condition, the participants had no additional secondary task requirements. In the easy condition, the participants simultaneously repeated aloud a single letter of the alphabet given to them at the beginning of each training trial. In the difficult condition, the participants simultaneously recited aloud the alphabet in backward order by every third letter (i.e., skip two letters), starting from a letter cue given to them at the beginning of each training trial (e.g., if the cue was "w," the participant was to say "w, t, q, n," etc.). The difficult task used here might appear to be unusual, but it requires the skill component of keeping track of where one is in a sequence of events. This component skill is a part of many everyday activities that involve duration production, such as when speakers need to judge how much time has elapsed and how much is remaining for their talk as they keep track of what they have already said and what should be said next.

Each training condition contained two different practice schedules (i.e., blocked and mixed). For the blocked schedule, the 6 trials of a given interval occurred successively. For the mixed schedule, a 6-trial block included 1 of each interval. During transfer, there were 12 trials in a block following the mixed arrangement. No secondary tasks were used during transfer in any of the conditions. The order of intervals was random in the blocked schedule and was random within each block in the mixed schedule and in the transfer blocks. The order of presentation of the blocks for each condition during training was reversed for half of the participants in each condition, yielding a total of 12 counterbalancing subgroups. All the participants received exactly the same transfer test.
There were three dependent measures. (1) The proportional absolute error is the absolute (i.e., unsigned) difference between the interval produced and the specified interval, divided by the specified interval [i.e., (| produced interval - specified interval|)/specified interval], which gives a normalized assessment of error magnitude. (2) The proportional relative error is the signed difference between the produced interval and the specified interval, divided by the specified interval (i.e., a signed proportional difference). This measure is just like the earlier one but uses signed values, rather than absolute values. It provides an index of response bias. When the produced interval is longer than the specified interval, there is positive bias by this index, whereas when the produced interval is shorter than the specified interval, there is negative bias. (3) The index of variation used is the root mean square deviation (RMSD), which is calculated with respect to the participants' average production for a given interval position. For the proportional relative error measure, we will include only an analysis of the training data, not of the transfer data, because for the proportional relative error both positive and negative values reflect erroneous responses, so that mean values of this measure do not provide a clear assessment of error magnitude. For a similar reason, only the training data will be reported for the RMSD measure.

In summary, the design for the training trials was a $3 \times 2 \times 6$ mixed factorial, with training condition (control, easy, or difficult) and training schedule (blocked or mixed) as between-subjects variables and interval position (first, second, third, fourth, fifth, or sixth occurrence of a given interval) as a within-subjects variable. Note that in the blocked training schedule, interval position corresponded to occurrences of a given interval on successive trials within a single block. In contrast, in the mixed training schedule, interval position corresponded to occurrences of a given interval on successive blocks. For the analysis of proportional relative error, an additional within-subjects variable was included: interval magnitude (short or long). The analysis of the transfer data employed a $3 \times 2 \times 3$ factorial design, with training condition and training schedule as between-subjects variables and interval type (old actual, new within, or new outside) as a within-subjects variable.

\section{Participants}

Thirty-six undergraduate college students participated for credit in an introductory psychology course. The participants were assigned by a fixed rotation to the 12 counterbalancing training subgroups, with 12 participants in each training condition, 6 in each training schedule, and 3 in each counterbalancing subgroup.

\section{Materials and Apparatus}

Macintosh computers were used for the training and transfer tests. The program was written using the PsyScope application (Cohen, MacWhinney, Flatt, \& Provost, 1993). The fixed intervals of time used in the training phase were chosen from within a range of 15-45 sec, with three of the intervals fixed below $30 \mathrm{sec}$ (i.e., 38.314 units) and three of the intervals fixed above $30 \mathrm{sec}$. The 30-sec cutoff between short and long interval magnitudes was arbitrary. Specifically, training intervals below $30 \mathrm{sec}$ (i.e., short intervals) were 21,25 , and 32 units. Training intervals above $30 \mathrm{sec}$ (i.e., long intervals) were 47, 50, and 56 units. These values were arbitrarily chosen with the intention of providing a balanced representation of the practice range but unequal spacing between intervals and a lack of symmetry in the distribution of intervals, so as to preclude easy extrapolation from one interval to others. The fixed intervals of time used in the transfer phase included some actually used in training and some untrained, which were either within or outside the practice range. The transfer intervals, like the training intervals, were unequally spaced and asymmetrical. Specifically, the transfer intervals were $12,19,21,23,32,36,43,47,54,56,58$, and 67 units. 
For the secondary task conditions, the letter cue was randomly selected from the alphabet and presented at the beginning of each training trial. Also, cassette tape recorders were used to document that each participant was attempting to say aloud the appropriate letter sequence in the secondary task conditions.

\section{Procedure}

During the training phase of the experiment, the participants were given feedback on the actual length of the interval that they had produced and on the difference between their produced interval and the specified interval, both in the arbitrary units. No feedback was given during the transfer phase of the experiment.

The participants were individually tested in a quiet room, free from outside distractions. All the participants were asked to remove their watches prior to beginning the experiment. The instructions for the training trials were presented on the computer screen and were as follows:

You will be asked to estimate a certain interval of time, expressed in fixed arbitrary "units" (not standard units like seconds or minutes). At the beginning of each trial, you will be told the number of units in the interval for you to estimate. Next you will hear a beep. Then when you're ready, press the space bar to indicate that the specified number of units have passed since the beep.... You will be given feedback at the end of each trial on the length of the interval in units that you estimated and the difference between that estimated interval and the specified interval. Your task is to try to become as accurate as you can in estimating these time intervals.

Additional instructions were given for the secondary tasks. Specifically, for the easy condition, the participants were told that, along with the task of producing a certain interval of time, they were to repeat aloud simultaneously and at their own pace the letter cue presented to them. The participants in the difficult condition were told to recite aloud, simultaneously with the primary task, the alphabet in backward order by every third letter, beginning from (and including) the letter cue presented to them, again at their own pace. They were further instructed that if they reached the beginning of the alphabet, they were to revert to the end of the alphabet and continue from that point. After reviewing the instructions with each participant in the secondary task conditions, a practice trial of the appropriate secondary task was given to each participant to ensure that the instructions were understood. Each participant in the easy condition was instructed to practice for the experimenter the task of repeating aloud the single letter "J." To ensure that the participants could perform the task correctly, each participant in the difficult condition was instructed to practice for the experimenter the task of reciting aloud the alphabet in backward order by every third letter, beginning from the letter "J," and was allowed to continue to do so until he or she reached the letter "U." Accuracy of the sequence of letters spoken aloud during the experiment was not examined, however. There was no feedback given for accuracy on either secondary task, except during the instruction phase before the experiment began.

After completing the training phase, the participants were given instructions for the transfer phase, which did not include any secondary tasks or any feedback. After that phase, they were given a questionnaire asking them how they had produced the number of units in the experiment. The participants in the secondary task conditions were subsequently asked whether they thought that the secondary task had made producing the number of units easier or harder and then were requested to explain their answer to this question.

\section{Results}

\section{Training}

For the analysis of training, as well as for all subsequent analyses, we will report all the significant $(p<.05)$ main effects and interactions, and we will discuss those that are not significant only when they are of theoretical interest.
Proportional absolute error. In the training phase, the proportional absolute error on the primary task of duration production was affected by the existence and nature of the secondary task $\left[F(2,30)=6.85, p=.004 ; \eta^{2}=\right.$ $.31]$. According to pairwise comparisons ( $t$ tests) with a Bonferroni correction ( $p<.017$; see, e.g., Howell, 1989), this effect reflects worse overall performance in the difficult condition (.223) than in the easy condition (.100). The control condition (.147) was intermediate and did not differ from either of the other conditions. In the easy condition, the repetitive, rhythmic nature of the secondary task may, in fact, have assisted in applying a counting strategy, despite the conflicting articulation requirement.

There was no overall statistically significant difference between blocked (.168) and mixed (.145) training schedules. An improvement in accuracy for the producing of durations was evident across trials of a given interval; the main effect of interval position was significant $\left[F(5,150)=16.83, p<.001 ; \eta^{2}=.36\right]$. A linear trend test was significant $[t(30)=-4.77, p<.001]$. According to pairwise comparisons ( $t$ tests) with a Bonferroni correction $(p<.003)$, this effect reflects higher proportional absolute error for the first interval position, relative to all subsequent interval positions, which did not differ from each other (first, .278; second, .159; third, .137; fourth, .136; fifth, .118; sixth, .115), suggesting that most of the learning occurred during the first interval position. The same pattern was found for all three conditions; the interaction of training condition and interval position was not significant.

Proportional relative error. There was a significant main effect of interval magnitude on the proportional relative error $\left[F(1,30)=12.58, p=.002 ; \eta^{2}=.30\right]$, with shorter interval magnitudes showing positive bias (.024) and longer interval magnitudes showing negative bias $(-.058)$. A two-way interaction between interval magnitude and interval position was significant $[F(5,150)=$ $\left.3.44, p=.006 ; \eta^{2}=.10\right]$. This two-way interaction was qualified by a significant three-way interaction between training condition, interval magnitude, and interval position $\left[F(10,150)=2.01, p=.036 ; \eta^{2}=.12\right]$. The participants in the control and easy conditions showed a trend toward a negative bias for long interval magnitudes and no bias for short interval magnitudes. In contrast, the participants in the difficult condition showed a trend toward a negative bias for long interval magnitudes and a trend toward a positive bias for short interval magnitudes. The participants generally showed less error as they progressed through successive trials for a given interval, indicating an increase in the knowledge necessary to produce time intervals over the full range. This effect was most prominent for the difficult condition (see Figure 1).

RMSD. The participants generally showed less variability as they progressed through successive trials for a given interval; the main effect of interval position was significant for RMSD [first, 12.323; second, 10.779; third, 10.051; fourth, 10.310; fifth, 10.172; sixth, 10.215; $\left.F(5,150)=3.00, p=.013 ; \eta^{2}=.09\right]$. A linear trend test was significant $[t(30)=-2.36, p=.025]$. In addition, 
RMSD was smaller for the mixed schedule (10.275), in which all trials of a given interval position occurred in the same block, than for the blocked schedule (11.008), in which trials of a given interval position occurred in different blocks; the main effect of training schedule was significant $\left[F(1,30)=4.49, p=.043 ; \eta^{2}=.13\right]$. This effect of training schedule can be explained simply by the fact that learning is greater across than within blocks.

\section{Transfer}

There was a significant main effect of training condition on proportional absolute error in the transfer test $\left[F(2,30)=15.70, p<.001 ; \eta^{2}=.51\right]$. According to pairwise comparisons ( $t$ tests) with a Bonferroni correction $(p<.017)$, this effect reflects worse performance in the difficult condition (.377) than in both the control condition (.106) and the easy condition (.126), which did not differ from each other. This disadvantage for the difficult condition occurred even though no secondary task was used during transfer in any condition. As in training, there was no overall difference between blocked (.231) and mixed (.175) training schedules in the transfer test. Furthermore, there was no effect of interval type during transfer (old actual, .202; new within, .201; new outside, $.206)$.
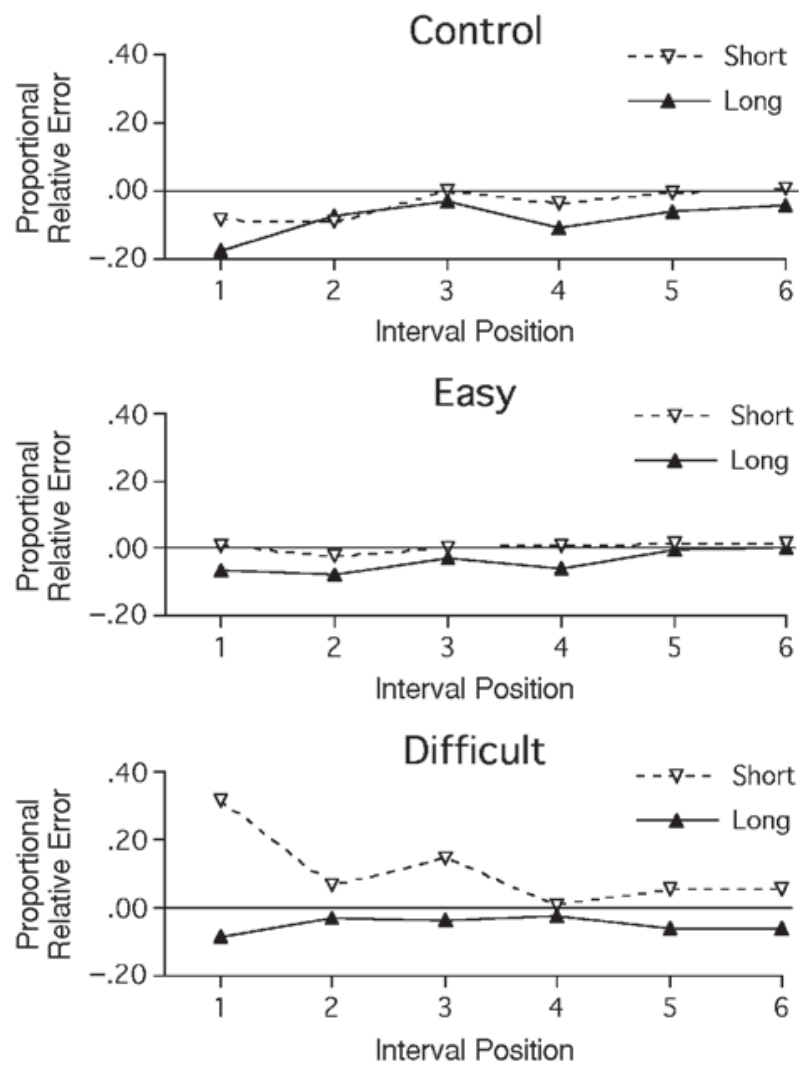

Figure 1. Mean proportional relative error as a function of training condition, interval magnitude, and interval position during training in Experiment 1. The horizontal lines indicate no bias (i.e., negative bias occurs below the line, and positive bias occurs above the line).
To examine further the effects of training condition on performance during transfer, we compared, post hoc, performance for the last interval position during training with performance overall during the transfer test (see Figure 2). This comparison yielded a significant interaction of training condition and session $[F(2,30)=6.48, p=$ $\left..005 ; \eta^{2}=.30\right]$, as well as main effects of both training condition $\left[F(2,30)=17.62, p<.001 ; \eta^{2}=.54\right]$ and session $\left[F(1,30)=15.64, p<.001 ; \eta^{2}=.34\right]$. This statisti$\mathrm{cal}$ analysis should be interpreted with caution, because of the numerous confounding differences between training and transfer (e.g., the intervals, the number of trials, the practice schedule, and the presence of feedback). Nevertheless, it indicates clearly that performance on the transfer test was worse than that on the last interval position during training for both the easy condition $[t(11)=2.50$, $p=.030]$ and the difficult condition $[t(11)=3.63, p=$ .004] (each of which involved a secondary task during training, but not during transfer), but not for the control condition (which did not involve any secondary task in either the training or the transfer phase). Removing the secondary task actually degraded performance for those participants trained with the secondary task. In fact, performance in the difficult condition during transfer was even worse (but not significantly so) than that in the control condition at the start of training, as can be seen in Figure 2, which also includes the first interval position during training.

\section{Retrospective Reports}

Two participants in the difficult condition failed to describe any strategy that they might have used in the task. Of the 34 remaining participants, 19 ( 8 in the control condition, 7 in the easy condition, and 4 in the difficult condition) specifically used the word count in their strategy description. An additional 7 participants (3 in the easy condition and 4 in the difficult condition) said that they had produced units in terms of some number of times that they had said the letters of the alphabet. Furthermore, 3 participants in the control condition said that they had used their sense of the length of a second to produce the units; 1 participant in the difficult condition had counted $1 \mathrm{sec}$ as equivalent to 3 units, and 1 participant in the easy condition had used one hand for fives and another for tens. Thus, 31 of the 34 responding participants clearly described some type of counting strategy. Of the remaining 3 participants, 1 in the control condition had used tapping, breathing, and guessing to produce units; 1 in the easy condition had produced units by comparing trials; and 1 in the difficult condition had just guessed.

In the easy condition, 10 of the 12 participants stated directly or implied in their answer that continuous repetition of a given letter had made counting easier, because the secondary task either had given them something to focus on or had given them something to count. Only 2 participants in this training condition specified that the secondary task had made duration production more difficult, because they had been unable to focus on both tasks simultaneously. We found similar results for the 10 partic- 


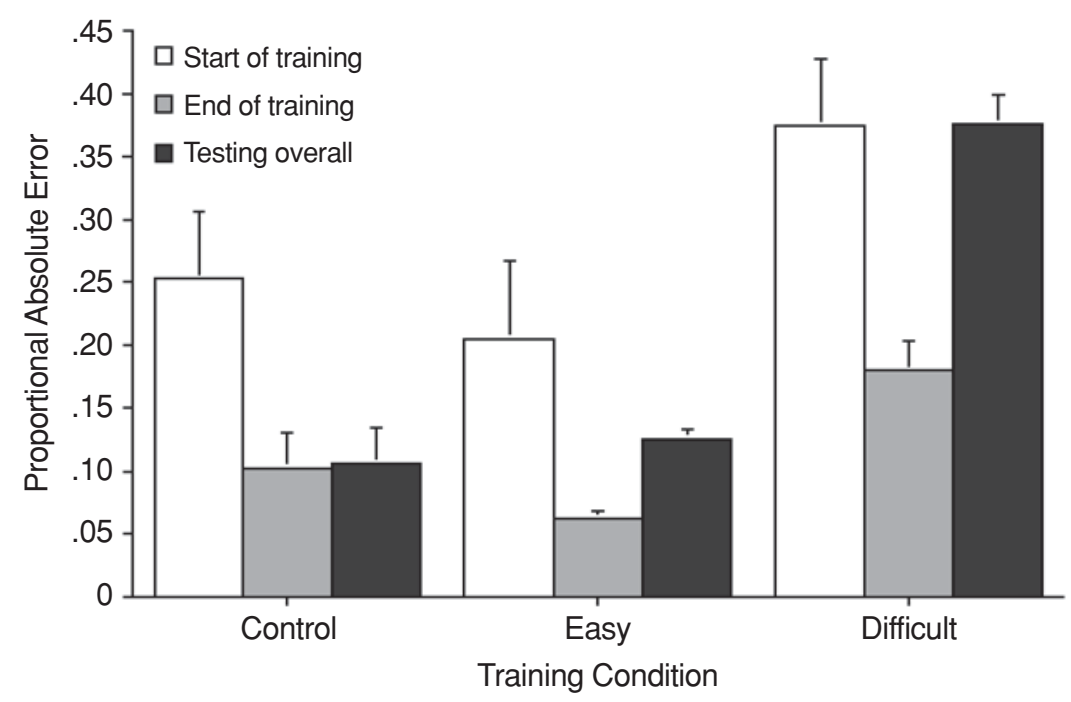

Figure 2. Mean proportional absolute error on the first interval position during training, the last interval position during training, and testing overall as a function of training condition in Experiment 1. Error bars show the positive standard error of the mean.

ipants who filled out the questionnaire from the difficult condition, with only 1 participant stating that the secondary task had made duration production more difficult and the other 9 stating that the secondary task had facilitated the producing of units.

\section{Discussion}

As was expected, duration production performance during training was more accurate under the easy secondary task than under the difficult secondary task, thereby verifying the effectiveness of the secondary task difficulty manipulation. More important, there was improvement during training across interval trials in terms of all three measures of performance: proportional absolute error, proportional relative error, and RMSD. For proportional absolute error, this improvement reflected an increase in production accuracy, whereas for proportional relative error the improvement took the form of decreasing bias (negative bias for long intervals and positive bias for short intervals) across interval trials, and for RMSD the improvement took the form of decreasing variability.

Improvement in producing intervals of time during training was not specific to the particular trained intervals but was specific to the secondary task employed. At transfer, the participants showed no greater error for new intervals outside the practice range than for new intervals within the practice range or even for old actual intervals, in agreement with the suggestion from the research of Fetterman and Killeen (1990) that a counting strategy can be maintained across a range of intervals. In contrast to this form of generalizability, the effects of changes in the secondary task indicated skill specificity. That is, the participants showed the highest proportion of errors during transfer when training included the difficult task, even though no secondary task was involved in the transfer test.
Furthermore, the participants were worse during transfer than at the end of training when the secondary task used during training was not required during transfer. Removing a secondary task, especially a very difficult one, actually disrupted performance on the primary task. These findings can be understood on the assumption that the same cognitive operations, based on the fixed fundamental time unit, are used for duration production of different target intervals but that the cognitive operations used for duration production differ with changes in the secondary task. It appears from the retrospective reports that, despite the conflicting articulation requirements of the secondary tasks, most of the participants reported using a form of counting in all three conditions. However, the particular counting strategy was not the same for all the conditions but, rather, depended on the secondary task. For example, the participants might have subvocalized a sequence of numerals in the control condition but might have, instead, kept track of the number of letters articulated in the difficult condition.

On the basis of previous work demonstrating contextual interference (e.g., Battig, 1972, 1979; Schneider et al., 2002; Schneider et al., 1995), we had predicted that a blocked practice schedule would yield superior performance during training but inferior performance during transfer, relative to a mixed practice schedule. No effects of practice schedule were found, however, for the proportional absolute error either during training or during transfer. Recall that the blocked and the mixed practice schedules concerned the arrangement of the produced intervals but not the arrangement of the secondary task conditions. This finding can be explained with the assumption that the same counting strategy is used to produce different intervals; the cognitive operations for duration production are affected only when the secondary task is changed. 
Another prediction, derived from the previous work on contextual interference (Schneider et al., 2002), was that training with the difficult alphabet secondary task would lead to superior performance during transfer, relative to training with either the easy secondary task or no secondary task. To the contrary, the opposite result was found; duration production errors were greater during transfer in the difficult training condition than in both the easy and the control training conditions. Because the cognitive operations used during training in the difficult condition could not be used during transfer, performance during transfer suffered, and no benefit could be derived from the difficulty encountered during training.

\section{EXPERIMENT 2}

The aim of Experiment 2 was to extend the findings in Experiment 1 concerning the effects of removing a secondary task on transfer and to investigate the long-term retention of the duration production skill. Specifically, in Experiment 2, we examined training and retraining of the time production skill after a 1-week delay. In both training and retraining, the participants were given six blocks of trials, with each block containing six different intervals, which were the same as those used during training in Experiment 1. In Session 1, half of the participants were trained under the difficult secondary task, and the remaining half under no secondary task (control). During retraining, 1 week later, half of the participants in each training condition were retrained under the difficult secondary task, and the remaining half under no secondary task.

Transfer testing in Experiment 1 was conducted with no secondary tasks. Thus, we could examine the effects of removing a secondary task on performance, but we could not examine the effects of adding a secondary task after training without a secondary task. In Experiment 2, each training condition was subdivided into different retraining groups so that all directions of transfer could be examined. To simplify the experimental design, we included only two training conditions in Experiment 2. Because the results of the difficult task were most clear-cut in Experiment 1 , we eliminated the easy condition and compared only the difficult condition with the control condition in Experiment 2. Also, to simplify the experimental design and because there was no support for the contextual interference hypothesis in Experiment 1, we removed the variable of training schedule. During both training and retraining, only mixed blocks of trials were employed; that is, each block included one trial with each interval.

In Experiment 1, performance was worse during transfer than for the last block of training in the difficult and easy conditions. However, as was discussed earlier, because of the confounding differences (e.g., in the intervals, the number of trials, the practice schedule, and the presence of feedback), no uncontaminated statistical comparison of performance during training and transfer could be conducted. To eliminate that problem in the present experiment, the confoundings were removed. Specifically, the same intervals, number of trials, practice schedule, and feedback were used during each block of training and retraining. On the basis of the findings in Experiment 1, we predicted that training would benefit performance at the start of retraining only when retraining involved the same requirements as training (i.e., only when the secondary task was either present or absent during both sessions).

\section{Method}

\section{Design}

As in Experiment 1, the primary task involved learning to produce fixed intervals of time expressed in units, with 1 unit of time equal to $783 \mathrm{msec}$. The training intervals in Experiment 2 were the same as those in Experiment 1, and the same intervals were used during both training and retraining. Thus, in Experiment 2, each participant was trained with feedback for six blocks of six trials and then, after a 1-week delay, was retrained, again with feedback, for six additional blocks of six trials.

Also, in Experiment 2, we included two of the training conditions used in Experiment 1: difficult and control. Each training condition contained two retraining groups, same and switch, depending on whether or not retraining matched training. There were, thus, four subgroups: (1) same D-D (i.e., both training and retraining with the difficult task), (2) switch D-C (i.e., training with the difficult task and retraining with the control task), (3) same $\mathrm{C}-\mathrm{C}$ (i.e., both training and retraining with the control task), and (4) switch C-D (i.e., training with the control task and retraining with the difficult task). The dependent measures (proportional absolute error, proportional relative error, and RMSD) were the same as those used in Experiment 1 .

Only mixed blocks of trials were used in training and retraining. One of the two sequences of trials used with mixed blocks in Experiment 1 during training was used here for training, and the other sequence (the reverse) was used for retraining.

In summary, the overall design for training was a $2 \times 6$ mixed factorial, with training condition (control or difficult) as a betweensubjects variable and block (first, second, third, fourth, fifth, or sixth) as a within-subjects variable. As in Experiment 1, for the analysis of proportional relative error, the additional variable of interval magnitude (short or long) was included. In addition, a set of analyses was conducted that focused on retention and transfer. This set was limited to the last block of training and the first block of retraining. The same factors were included in this set as in the analysis of training, except that the factor of block was replaced by the factor of session (first or second) and there was the additional betweensubjects variable of retraining group (same or switch).

\section{Participants}

Forty-eight undergraduate college students participated for credit in an introductory psychology course. The participants were assigned by a fixed rotation to the four subgroups, with 24 participants in each training condition (control or difficult) and 12 in each subgroup (same D-D, switch D-C, same C-C, or switch C-D).

\section{Materials and Apparatus}

The materials and apparatus in Experiment 2 were identical to those in Experiment 1.

\section{Procedure}

The procedure in Experiment 2 was identical to that in Experiment 1 with the same conditions. When the participants returned for retraining, they were told by the experimenter that the secondary task would be the same as that during training (same) or that the secondary task would be different (switch). In both cases, at the start of the session, the participants were shown on the computer screen the full set of instructions appropriate for their task. The participants filled out the retrospective questionnaire at the end of each session. 


\section{Training}

\section{Results}

Proportional absolute error. Performance improved significantly across blocks $[F(5,230)=22.18, p<.001$; $\left.\eta^{2}=.33\right]$. A linear trend test was significant $[t(46)=$ $-5.31, p<.001]$. According to pairwise comparisons $(t$ tests) with a Bonferroni correction $(p<.003)$, this effect reflects an improvement from the first block (.260) to the subsequent blocks, which did not differ from each other (Block 2, .161; Block 3, .157; Block 4, .142; Block 5, .140; Block 6, .155).

Training with the difficult secondary task led to higher error than did training with the control task (difficult, .211; control, .127); the main effect of training condition was significant $\left[F(1,46)=18.68, p<.001 ; \eta^{2}=.29\right]$.

Proportional relative error. The results for the proportional relative error are summarized in Figure 3 as a function of block, interval magnitude, and training condition. As was also found in Experiment 1, improvement in the producing of intervals reflected a decreasing bias to produce intervals toward the central tendency of the practice range. Thus, there was a negative bias for the long intervals $(-.071)$ and, to a lesser degree, a positive bias for the short intervals (.036), especially at the beginning of practice. There was a significant interaction of interval magnitude and block $[F(5,230)=2.29, p=.047$; $\left.\eta^{2}=.05\right]$, as well as a main effect of interval magnitude $\left[F(1,46)=50.86, p<.001 ; \eta^{2}=.53\right]$. In addition, there was a significant interaction of interval magnitude and training condition $\left[F(1,46)=11.30, p=.002 ; \eta^{2}=.20\right]$, reflecting the fact that the positive bias for short intervals was greater in the difficult training condition (.064) than in the control training condition (.008) and, similarly, the negative bias for long intervals tended to be greater in the difficult training condition $(-.093)$ than in the control training condition $(-.048)$. That is, the participants in the difficult training condition showed worse performance as measured by proportional relative error, as well as proportional absolute error, than did the participants in the control training condition.

RMSD. As in Experiment 1, the participants generally showed less variability as they progressed through successive blocks; the main effect of block was significant for RMSD [first, 12.272; second, 10.660; third, 10.821; fourth, 10.216; fifth, 11.830 ; sixth, $11.342 ; F(5,230)=$ $\left.3.82, p=.002 ; \eta^{2}=.08\right]$.
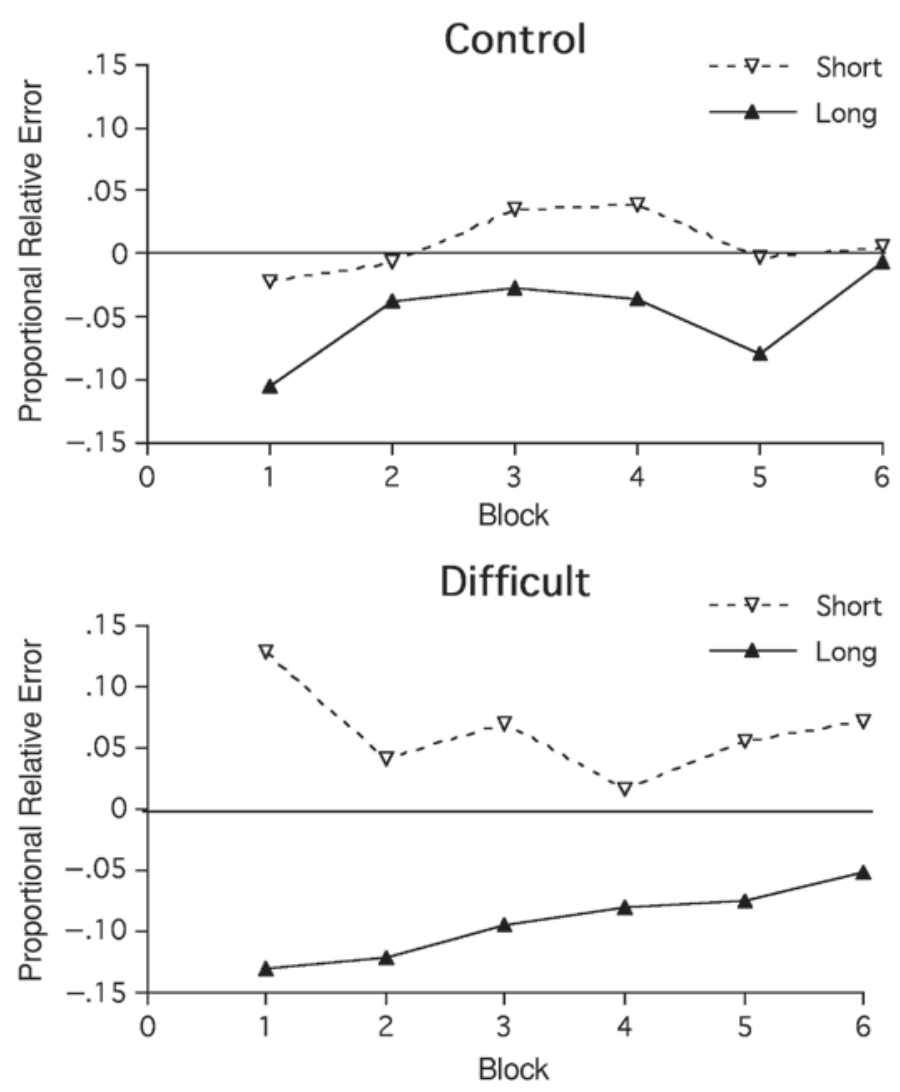

Figure 3. Mean proportional relative error as a function of block, interval magnitude, and training condition during training in Experiment 2. The horizontal lines indicate no bias (i.e., negative bias occurs below the line, and positive bias occurs above the line). 


\section{Retention and Transfer}

To examine retention and transfer, we focused on the two blocks surrounding the 1-week retention interval: Session 1, Block 6 and Session 2, Block 1. The results for retention and transfer are summarized in Figure 4 in terms of the mean proportional absolute error on those blocks as a function of retraining group and training condition.

The participants who performed the same task in both sessions showed no change in absolute error across the two sessions (Session 1, .166; Session 2, .167) despite the 1 -week delay separating them. This finding demonstrates remarkable skill durability. In contrast, the participants who switched tasks showed a dramatic increase in error across the two sessions (Session 1, .144; Session 2, .244), demonstrating remarkable skill specificity. This observation is supported by a significant interaction of retraining group and session $\left[F(1,44)=11.59, p=.001 ; \eta^{2}=.21\right]$, as well as a main effect of session $[F(1,44)=11.78, p=$ $\left..001 ; \eta^{2}=.21\right]$.

The lack of change between sessions for the same retraining group was evident both for the control training condition (same $\mathrm{C}-\mathrm{C}$ ) and for the difficult training condition (same D-D). Likewise, the increase in error between sessions for the switch retraining group occurred for both training conditions (i.e., for both switching directions), but the increase was much greater when the participants switched from the control condition to the difficult condition (switch C-D) than when they switched from the difficult condition to the control condition (switch $\mathrm{D}-\mathrm{C}$ ). Consequently, the three-way interaction of retraining group, training condition, and session was significant
$\left[F(1,44)=6.08, p=.018 ; \eta^{2}=.12\right]$, as was the interaction of training condition and session $[F(1,44)=6.27$, $\left.p=.016 ; \eta^{2}=.12\right]$.

Figure 4 also includes the results for the first block of training during Session 1, as well as for the last block of retraining during Session 2. These blocks were not included in the statistical analysis but provide useful points of comparison. For example, the performance level for the switch D-C group during Session 2, Block 1 was equivalent to that for both the switch C-D group and the same $\mathrm{C}-\mathrm{C}$ group during Session 1, Block 1. Thus, despite the great improvement evident from Block 1 to Block 6 in Session 1 for the switch D-C group, that group did not benefit at all from that improvement when it switched from the more difficult secondary task in Session 1 to the simpler control task in Session 2. A parallel set of results was found by comparing performance for the switch C-D group on Session 2, Block 1 with performance for the switch D-C and same D-D groups on Session 1, Block 1. To document these patterns statistically, we computed an index of transfer by subtracting performance on Session 2, Block 1 in each condition from the average performance for the same task on Session 1, Block 1 across all the participants given that task. Thus, for example, the transfer index for the switch D-C group was computed by subtracting performance for the control task on Session 2, Block 1 from the average performance on Session 1, Block 1 across the participants in the same $\mathrm{C}-\mathrm{C}$ and switch $\mathrm{C}-\mathrm{D}$ groups who were given that task initially. With a $t$ test, this index was compared with 0 for each condition separately. No transfer was evident in either switch

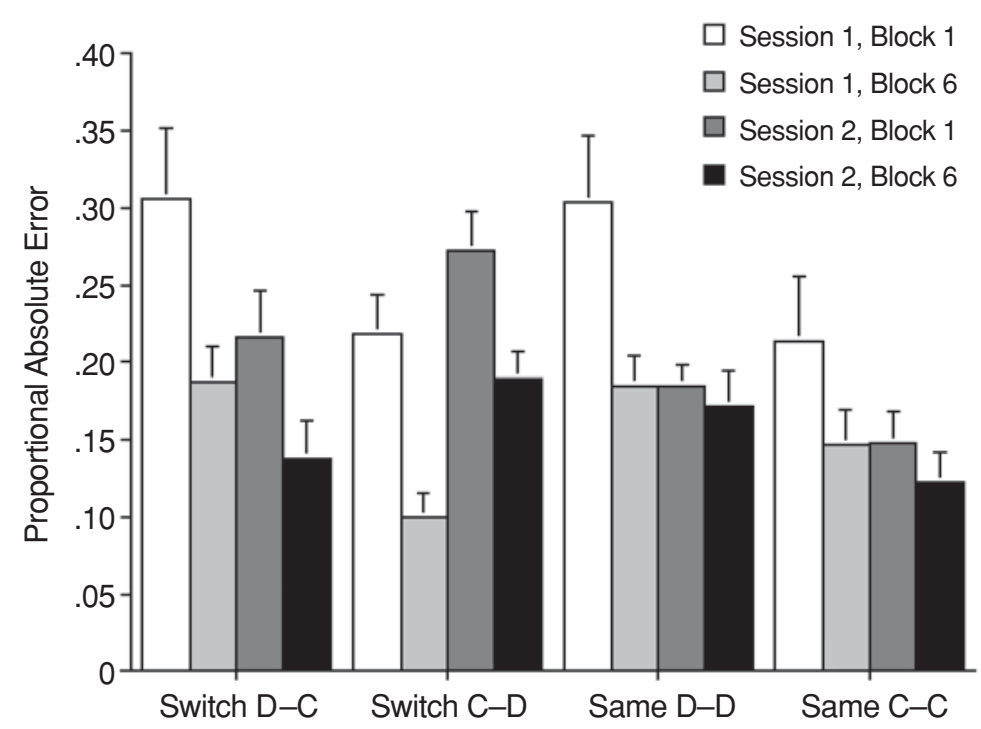

Figure 4. Mean proportional absolute error on the first block of training (Session 1, Block 1), the last block of training (Session 1, Block 6), the first block of retraining (Session 2, Block 1), and the last block of retraining (Session 2, Block 6) as a function of retraining group and training condition in Experiment 2. D, difficult task; $C$, control task. The first letter represents the training condition, and the second letter represents the secondary task used during retraining. Error bars show the positive standard error of the mean. 
retraining group; the mean transfer index was 0.0004 for the switch D-C group $[t(11)=0.01, p=.989]$ and was 0.03 for the switch $\mathrm{C}-\mathrm{D}$ group $[t(11)=1.23, p=.246]$. In contrast, large transfer was evident for both same retraining groups by the same measure; the mean transfer index was 0.07 for the same $\mathrm{C}-\mathrm{C}$ group $[t(11)=3.46$, $p=.005]$ and was 0.12 for the same D-D group $[t(11)=$ 9.30, $p<.001]$.

Another, possibly more sensitive way to examine transfer is to assess savings in relearning (see, e.g., Kehoe \& Holt, 1984; Weidemann \& Kehoe, 2003). For the switch retraining groups, was there evidence for savings in relearning across all six blocks in Session 2, relative to learning across all six blocks of Session 1, despite the fact that there was no evidence for improved performance on the first block of Session 2, relative to the first block of Session 1 ? To answer this question, we conducted an analysis involving all six blocks during each session but restricted to the two switch retraining groups $(\mathrm{C}-\mathrm{D}$ and $\mathrm{D}-\mathrm{C})$. That analysis revealed a significant main effect of block $\left[F(5,110)=15.62, p<.001 ; \eta^{2}=.42\right]$ but no two-way interaction of session and block or three-way interaction of training condition, session, and block. Therefore, there was no evidence that the improvement across blocks for the two switch groups was larger in Session 2 (Block 1, .244; Block 2, .152; Block 3, .177; Block 4, .139; Block 5, .163; Block 6, .164) than in Session 1 (Block 1, .262; Block 2, .167; Block 3, .161; Block 4, .140; Block 5, .147 ; Block $6, .144)$. Thus, no savings was evident, and the numerical trend showed the opposite pattern, suggesting, if anything, less rapid learning in Session 2 than in Session 1.

By comparing the first block of retraining with the last block of retraining for each group (Session 2, Block 1 and Session 2, Block 6) in Figure 4, it is also clear that improvement occurred during retraining overall, especially for the switch groups, who changed to a different task during retraining. In fact, the switch groups ultimately (after a full session of retraining) reached approximately the same level of performance on their tasks as did the two groups who did not change tasks.

\section{Retrospective Reports}

On the retrospective questionnaire for Session 1, 20 of the 48 participants ( 4 in the difficult training condition and 16 in the control training condition) specifically used the word count in describing the strategy that they had used. An additional 8 participants in the difficult training condition said that they had kept track of the number of times that they had said letters to produce units. Furthermore, 3 participants ( 1 in the difficult training condition and 2 in the control training condition) said that they had used seconds to produce units, and 1 participant in the control training condition said that she had paused "after every three numbers." Thus, 32 of the 48 participants clearly described a counting strategy for Session 1. Of the remaining 16 participants, 2 in the control training condition said that they had used tapping, 1 in the control train- ing condition said that she had used a hand pattern, and 13 (11 in the difficult training condition and 2 in the control training condition) said that they had simply guessed.

On the retrospective questionnaire for Session 2, 22 of the 48 participants ( 4 given the difficult task and 18 given the control task in that session) specifically used the word count in describing the strategy that they had used. Seven other participants given the difficult task said that they had kept track of the number of times that they had said letters to produce units. Furthermore, 1 participant given the control task said that he had used seconds to produce units. Thus, 30 of the 48 participants clearly described a counting strategy for Session 2. Of the remaining 18 participants, 3 ( 2 given the control task and 1 given the difficult task) said that they had used tapping, 1 given the difficult task said that he had had an "awareness of time," and 14 (11 given the difficult task and 3 given the control task) said that they had simply guessed.

For Session 1, 8 of the 24 participants in the difficult training condition stated directly that the secondary task had made counting easier, because it either had given them something to focus on or had given them something to count. The other 16 participants in this training condition specified that the secondary task had made duration production more difficult, because they had been unable to focus on both tasks simultaneously (1 of these participants said that she had not been able to successfully perform the difficult secondary task). We found similar results for Session 2, with 6 participants stating that the difficult secondary task had made production easier and the remaining 18 stating that the secondary task had made the producing of time units more difficult.

\section{Discussion}

Duration production performance, in terms of both proportional absolute error and proportional relative error, was worse for the difficult training condition, relative to the control condition, verifying once more the effectiveness of the secondary task manipulation. Also, as in Experiment 1, there was general improvement during training across blocks in terms of all three measures of performance: proportional absolute error, proportional relative error, and RMSD.

In Experiment 1, a change in the secondary task requirements during transfer degraded performance on the primary task of duration production. However, only one type of change was examined-namely, elimination of the secondary task. In the present experiment, the effects both of adding and of deleting a secondary task were examined, and it was found that both types of changes degraded performance. In fact, for the participants whose secondary task requirements were altered, performance at the start of Session 2 was equivalent to that under the same secondary task conditions at the start of Session 1. Thus, there was no transfer of the primary duration production skill under changing conditions, despite the fact that there was significant improvement during training and despite the fact that retention across the same delay 
interval was perfect for the participants whose secondary task requirements were not altered. Together, these findings provide strong evidence for specificity of training. There were benefits of training on performance during retraining only when the cognitive operations acquired during training could be used during retraining.

A post hoc analysis of performance on the secondary alphabet task showed a nonsignificant tendency toward improvement across blocks of trials during training in both the accuracy and the rate of performing that task (see the Appendix). Along with a significant increase in accuracy on the primary duration production task, this finding suggests that there was no trade-off in performance between the primary and the secondary tasks, despite the fact that primary task performance suffered when the secondary alphabet task was required and despite the suggestion from a correlation analysis that individuals who performed the secondary task at a faster rate tended to be less accurate on the primary task (again, see the Appendix). Instead of trading off one task for another, it appears from the retrospective reports that at least a sizable number of the participants developed a strategy for the primary duration production task that made use of the requirements of the secondary alphabet task.

\section{GENERAL DISCUSSION}

We conducted two experiments on producing temporal durations in terms of fixed arbitrary units. A prospective production paradigm was used to train and test participants on the skill of duration production, focusing on durations under $1 \mathrm{~min}$.

\section{Improvement During Training}

The participants improved during training across interval trials in Experiment 1 and generally across blocks in Experiment 2 with respect to all three measures of performance that we examined: proportional absolute error (i.e., production accuracy), proportional relative error (i.e., response bias), and RMSD (i.e., response variability). Also, as was expected, in terms of both proportional absolute and proportional relative error, performance during training was worse in Experiment 1 for the difficult secondary task than for the easy secondary task and in Experiment 2 for the difficult condition than for the control condition.

\section{Specificity of Training}

On the basis of previous demonstrations of skill specificity and the many theoretical notions deriving from Thorndike's (1906) theory of identical elements (e.g., Healy \& Bourne, 1995; Kolers \& Roediger, 1984; Morris et al., 1977; Rickard \& Bourne, 1995; Singley \& Anderson, 1989; Tulving \& Thomson, 1973), specificity of transfer was expected for the present task of duration production. On the other hand, Fetterman and Killeen's (1990) finding that the same counting strategy can be applied to different time intervals in duration reproduction suggested that, instead, generalizability of training might be found in this case.
Counting strategies. At the outset of this study, following Killeen and Weiss (1987), we assumed that counting would be the preferred strategy for duration production, and following Healy and Nairne (1985), we assumed that counting would require subvocalization in order to maintain an accurate representation of the number of units and the pacing between those units. To test this hypothesis, Experiment 1 included three training conditions that varied in the secondary task required by the participants: control, easy, and difficult. Both the easy and the difficult tasks required the participants to say letters out loud, thereby presumably inhibiting subvocalization of counting responses (although some subvocalization of numbers might have been possible). No secondary tasks were used during the transfer phase after any of these training conditions. We found a large effect of training condition on accuracy during training, with worse performance for the difficult training condition than for the easy training condition. This finding was consistent with our prediction that this task would disrupt the usual counting strategy. We also found that performance during transfer (when no secondary task was required in any condition) was much worse in the difficult training condition than in the control training condition, which suggests that the strategies acquired during training, although general across production intervals, were specific to the secondary task. In support of this suggestion, we found that performance on the transfer test was worse than that on the last block of training for both the difficult and the easy training conditions, but not for the control training condition. Removing the secondary task impaired performance for those participants trained with the secondary task. This is a unique finding that is surprising in many respects but consistent with the suggestion concerning the specificity of cognitive operations, or counting strategies, learned by the participants during training.

Functional task principle. These results can be understood if the participants in fact developed a counting strategy during training that differed between secondary tasks and depended on the requirements of the secondary task. In fact, the retrospective reports suggested that many of the participants were able to merge the primary duration production and secondary task requirements into a single functional task that incorporated and integrated components of both tasks. For example, some participants reported that the secondary task was helpful in duration production because it gave them something to count. Thus, the procedures that the participants used in the combined task appear to be different from those that they used in the primary task when it occurred in isolation, although a form of counting may have been involved in both. We shall refer to this observation as the functional task principle.

Transfer and retention. Experiment 2 provided a confirmation of the findings in Experiment 1 concerning the effects of a secondary task and supplied new information about the long-term retention of the duration production skill. Both training and retraining after a 1week delay were examined with both the control and the 
difficult secondary tasks. This experiment demonstrated clearly that improvement in producing intervals during training was completely specific to the condition (i.e., combination of primary and secondary tasks) used during training. The participants in both training conditions showed considerable improvement during training. When the participants from the two training conditions returned after a 1-week delay, they showed perfect retention for the duration production skill that they had acquired if they performed the same task during training and retraining. These results are in accordance with previous findings that knowledge and skill durability is high when the procedures are maintained across practice and test situations (e.g., Healy et al., 1992). However, when their secondary task condition was switched 1 week later during retraining from the task used during training, the participants showed a significant drop in performance. This drop was larger when they trained on the control task and retrained on the difficult task (switch C-D), but it occurred to some extent even when they trained on the difficult task and retrained on the control task (switch D-C). In fact, in both cases, performance at the start of retraining was equivalent to that for the same secondary task condition at the start of training. Thus, there was no forgetting across the 1-week delay when the secondary task conditions were the same and no transfer across the same delay when the secondary task conditions were switched. Note that there was a suggestion in Experiment 1 that removing a secondary task interfered with performance on the primary task. However, in Experiment 2, although performance in the switch retraining group declined across the retention interval, especially when the participants switched to a more difficult task (switch C-D), the level of performance at the start of retraining was not worse than that at the start of training, even when, as in Experiment 1, the participants switched to a less difficult task (switch D-C). Thus, changing secondary tasks does not necessarily interfere with primary task performance. However, finding no interference does not undermine the findings that there was no transfer when the secondary task was either added or removed and that there was no savings of relearning in Session 2, relative to learning in Session 1. Even in Experiment 2, when a secondary task was used during training but removed during retraining, performance at the start of retraining was equivalent to that at the start of training on the same task.

Most of the improvement during training occurred in the first block. That result might imply that the participants simply had learned in that block that a unit was approximately three fourths of a second (although no participant specifically mentioned that fact in the retrospective reports). Given this implication, it would not be surprising that the participants could show perfect retention over the 1-week delay when the secondary task condition remained the same, as was indeed the case. However, that implication would also lead to the prediction that the participants would show the benefit of this knowledge concerning the approximate value of the unit when the secondary task condition was switched. In fact, though, there was no evi- dence that the participants benefited from any previous learning in the switch retraining group when the secondary task was either added or removed. Thus, what the participants learned during the first block of training was not just the approximate value of a unit but, rather, something specific to the secondary task condition, such as a particular counting strategy or a set of cognitive operations.

Despite the lack of transfer found when the secondary task conditions were changed across the 1-week delay interval, by the end of retraining, performance for the participants who switched secondary task conditions between sessions was equivalent to that for the participants who maintained the same secondary task condition across the two sessions. This finding indicates that the problem of no transfer can be overcome given sufficient additional practice.

The results of Experiment 2, like those of Experiment 1, are compatible with the functional task principle, under the assumption that the cognitive operations acquired by the participants were unique to each combination of primary (duration production) and secondary tasks. Thus, rather than trading off the primary and secondary task requirements, the participants treated each combination of primary and secondary tasks as a functionally integrated set of requirements and developed a strategy that was appropriate for those requirements. This argument, based on the functional task principle, is similar to the proposal by Rah, Reber, and Hsiao (2000; Hsiao \& Reber, 2001), who used a primary serial reaction time task in combination with a secondary tone-counting task. They argued that the participants in their study did not treat the procedures required of them as a dual task but, rather, treated the tonecounting task as a source of variation relevant to the serial reaction time task.

An alternative explanation for the lack of transfer of performance on the primary task across secondary tasks concerns the fact that the secondary tasks required different amounts of attentional resources, so that different amounts of attentional resources were available for duration production (see, e.g., Posner \& Boies, 1971). This explanation does not seem consistent with the finding that there was no trade-off between accuracy in the primary task and accuracy in the secondary task. Nevertheless, in the present study, we cannot fully distinguish between this alternative and our proposed functional task principle.

\section{Contextual Interference}

During training in Experiment 1, for the participants given blocked training, all six intervals within a given block were the same, whereas for the participants given mixed training, all six intervals were included in each block. Presumably because of greater learning across than within blocks, we found a smaller value of RMSD (i.e., response variability) during training for the mixed training schedule than during that for the blocked training schedule.

On the basis of the principle of contextual interference (e.g., Battig, 1972, 1979; Schneider et al., 2002; Schneider et al., 1995), we expected that the participants given mixed 
training would perform worse during training but better on the transfer test than would the participants given blocked training. Contrary to these predictions, however, we found no difference between blocked and mixed training during either training or transfer for the measure of proportional absolute error. This outcome can be understood in terms of the counting strategy the participants reported having used to produce intervals. The lack of difference between blocked and mixed practice schedules would be expected if the participants had used the same counting strategy for producing all intervals, regardless of their length or their presentation order, as is suggested by the results of Fetterman and Killeen (1990). This shared counting strategy is also consistent with the finding in Experiment 1 of no difference in the accuracy of productions given during transfer testing to old intervals used during training and new intervals not previously trained, even new intervals outside of the practice range. Another way to view the finding concerning practice schedule is in terms of the fact that interval length was the blocked or mixed variable during training, which is probably not a factor that influences the cognitive operations employed on a trial. If the practice schedule manipulation had been based on the secondary task employed, instead of on the interval, a contextual interference effect might have been evident, because of the likely influence of the secondary task on the cognitive operations used for duration production.

Although the practice schedule manipulation did not involve the secondary tasks in Experiment 1, a test of the more general training difficulty hypothesis (Schneider et al., 2002), which was derived from the contextual interference principle, was provided by the manipulation of secondary tasks in the same experiment, because those tasks differed in difficulty. In particular, according to this more general hypothesis, it was predicted that duration production accuracy during transfer would be best when the training conditions had been most difficult. However, there was no support for this hypothesis, because duration production accuracy was worse during transfer for the difficult training condition than for the easy and control training conditions. The explanation for this finding, in agreement with the functional task principle, is that not only did the secondary tasks make learning the primary task more difficult, but also they changed the nature of the cognitive operations used in the primary task.

\section{Conclusions}

Other recent studies have shown that under some conditions, there is virtually no cost for performing two tasks concurrently (e.g., Ahissar, Laiwand, \& Hochstein, 2001; Schumacher et al., 2001; for an alternative to the no-cost interpretation, see Byrne \& Anderson, 2001). We extended the findings from those studies by providing evidence that performance after training with a difficult concurrent task may be better when that task is present than when that task is removed. In theoretical terms, the present findings are consistent with the functional task principle, under the assumption that the cognitive operations used to produce durations differ as a function of any secondary task employed during the primary task of duration production, because the primary and secondary tasks are integrated together. As is suggested by the retrospective reports, the participants typically used a counting strategy, but that strategy usually involved number counting with the control task and letter counting with the easy and difficult tasks. For the participants given the difficult secondary task, the requirement to say the alphabet backward by threes became integral with the requirement to produce durations, despite the seemingly disparate nature of these tasks. These findings, thus, extend the evidence for specificity of training by demonstrating that it is found not only with respect to the cognitive operations involved in the primary task, but also with respect to those involved in an irrelevant but concurrent secondary task. They indeed suggest that for practiced participants, primary and secondary tasks are not always performed independently but, rather, may functionally merge, or become integrated, into a single more complex task. Therefore, it is important to distinguish between the nominal tasks that an experimenter might impose (including separate primary and secondary task requirements) and the actual (i.e., functional) task that the participant masters (including integrated combinations of the primary and secondary task requirements). This conclusion, which we have called the functional task principle and is consistent with the arguments of Hsiao and Reber (2001), implies that what a participant learns in an experiment might not correspond exactly to the experimenter's conception of task requirements.

In practical terms, these findings highlight the importance of training individuals with the same operations that they will encounter subsequently, including irrelevant secondary operations, not just operations involved in the primary task. If the operations are changed, the individuals may return to initial levels of performance, so that training might be completely ineffective. Therefore, when a training regime is developed that will effectively prepare individuals for future performance in a task, it is important to take into consideration, to the greatest extent possible, the complete set of eventual task requirements. This recommendation may at first appear to coincide with common practice. However, in fact, there are many training situations that purposely violate this advice. To give just one example, competitive swimmers often wear "drag suits" during training (e.g., one bathing suit on top of another, which has the result of increasing resistance in the water) but then switch during competition to suits that minimize drag. Thus, the swimmers are violating the specificity-oftraining recommendation, because they may not be training using exactly the same operations as those that they will use during competition (i.e., the movements may be subtly changed). There is no question that drag should be minimized during competition, but the question raised here is whether drag should also be minimized, instead of increased, during training. The present study challenges the current practice and suggests, instead, that training with extra drag, even though it may promote strengthening or endurance, may not be optimal, so that performance 
during competition may suffer accordingly. More generally, the present study challenges any training routine in which the conditions and requirements for practice are not matched to those used for eventual testing.

\section{REFERENCES}

Ahissar, M., Laiwand, R., \& Hochstein, S. (2001). Attentional demands following perceptual skill training. Psychological Science, 12, 56-62.

Battig, W. F. (1972). Intratask interference as a source of facilitation in transfer and retention. In R. F. Thompson \& J. F. Voss (Eds.), Topics in learning and performance (pp. 131-159). New York: Academic Press.

BatTig, W. F. (1979). The flexibility of human memory. In L. S. Cermak \& F. I. M. Craik (Eds.), Levels of processing and human memory (pp. 23-44). Hillsdale, NJ: Erlbaum.

Byrne, M. D., \& Anderson, J. R. (2001). Serial modules in parallel: The psychological refractory period and perfect time-sharing. Psychological Review, 108, 847-869.

Clawson, D. M., King, C. L., Healy, A. F., \& Ericsson, K. A. (1995). Training and retention of the classic Stroop task: Specificity of practice effects. In A. F. Healy \& L. E. Bourne, Jr. (Eds.), Learning and memory of knowledge and skills: Durability and specificity (pp. 234 254). Thousand Oaks, CA: Sage.

Cohen, J. D., MacWhinney, B., Flatt, M., \& Provost, J. (1993). PsyScope: An interactive graphics system for designing and controlling experiments in the psychology laboratory using Macintosh computers. Behavior Research Methods, Instruments, \& Computers, 25, 257-271.

Fetterman, J. G., \& Killeen, P. R. (1990). A componential analysis of pacemaker-counter timing systems. Journal of Experimental Psychology: Human Perception \& Performance, 16, 766-780.

Healy, A. F., \& Bourne, L. E., Jr. (1995). Preface: Durability and specificity of knowledge and skills. In A. F. Healy \& L. E. Bourne, Jr. (Eds.), Learning and memory of knowledge and skills: Durability and specificity (pp. ix-xiv). Thousand Oaks, CA: Sage.

Healy, A. F., Fendrich, D. W., Crutcher, R. J., Wittman, W. T., Gesi, A. T., Ericsson, K. A., \& Bourne, L. E., Jr. (1992). The longterm retention of skills. In A. F. Healy, S. M. Kosslyn, \& R. M. Shiffrin (Eds.), From learning processes to cognitive processes: Essays in honor of William K. Estes (Vol. 2, pp. 87-118). Hillsdale, NJ: Erlbaum.

Healy, A. F., \& NaIrne, J. S. (1985). Short-term memory processes in counting. Cognitive Psychology, 17, 417-444.

Hicks, R. E., Miller, G. W., \& Kinsbourne, M. (1976). Prospective and retrospective judgments of time as a function of amount of information processed. American Journal of Psychology, 89, 719-730.

Howell, D. C. (1989). Fundamental statistics for the behavioral sciences (2nd ed.). Boston: PWS-Kent.

Hsiao, A. T., \& Reber, A. S. (2001). The dual-task SRT procedure: Fine-tuning the timing. Psychonomic Bulletin \& Review, 8, 336-342.

Kenoe, E. J., \& Holt, P. E. (1984). Transfer across CS-US intervals and sensory modalities in classical conditioning of the rabbit. Animal Learning \& Behavior, 12, 122-128.
Killeen, P. R., \& Weiss, N. A. (1987). Optimal timing and the Weber function. Psychological Review, 94, 455-468.

Kolers, P. A., \& Roediger, H. L., III (1984). Procedures of mind. Journal of Verbal Learning \& Verbal Behavior, 23, 425-449.

Morris, C. D., Bransford, J. D., \& Franks, J. J. (1977). Levels of processing versus transfer appropriate processing. Journal of Verbal Learning \& Verbal Behavior, 16, 519-533.

Parker, J. T., Healy, A. F., \& Bourne, L. E., JR. (2000, November). Training and transfer of duration estimation: The procedural reinstatement framework. Paper presented at the 41st Annual Meeting of the Psychonomic Society, New Orleans.

Posner, M. I., \& BoIEs, S. J. (1971). Components of attention. Psychological Review, 78, 391-408.

Rah, S. K.-Y., Reber, A. S., \& Hsiao, A. T. (2000). Another wrinkle on the dual-task SRT experiment: It's probably not dual task. Psychonomic Bulletin \& Review, 7, 309-313.

Rickard, T. C., \& Bourne, L. E., JR. (1995). An identical-elements model of basic arithmetic skills. In A. F. Healy \& L. E. Bourne, Jr. (Eds.), Learning and memory of knowledge and skills: Durability and specificity (pp. 255-281). Thousand Oaks, CA: Sage.

Rickard, T. C., Healy, A. F., \& Bourne, L. E., Jr. (1994). On the cognitive structure of basic arithmetic skills: Operation, order, and symbol transfer effects. Journal of Experimental Psychology, 20, 1139-1153.

SAWYER, T. F. (1999). Allocation of attention and practice in the production of time intervals. Perceptual \& Motor Skills, 89, 1047-1051.

Schneider, V. I., Healy, A. F., \& Bourne, L. E., Jr. (2002). What is learned under difficult conditions is hard to forget: Contextual interference effects in foreign vocabulary acquisition, retention, and transfer. Journal of Memory \& Language, 46, 419-440.

Schneider, V. I., Healy, A. F., Ericsson, K. A., \& Bourne, L. E., Jr. (1995). The effects of contextual interference on the acquisition and retention of logical rules. In A. F. Healy \& L. E. Bourne, Jr. (Eds.), Learning and memory of knowledge and skills: Durability and specificity (pp. 95-131). Thousand Oaks, CA: Sage.

Schumacher, E. H., Seymour, T. L., Glass, J. M., Fencsik, D. E., Lauber, E. J., Kieras, D. E., \& Meyer, D. E. (2001). Virtually perfect time sharing in dual-task performance: Uncorking the central cognitive bottleneck. Psychological Science, 12, 101-108.

Shea, J. B., \& Morgan, R. L. (1979). Contextual interference effects on the acquisition, retention, and transfer of a motor skill. Journal of Experimental Psychology: Human Learning \& Memory, 5, 179-187.

Singley, M. K., \& ANDERSON, J. R. (1989). The transfer of cognitive skill. Cambridge, MA: Harvard University Press.

THORNDIKE, E. L. (1906). The principles of teaching, based on psychology. New York: Seiler.

Tulving, E., \& Thomson, D. M. (1973). Encoding specificity and retrieval processes in episodic memory. Psychological Review, 80, 352-373.

Weidemann, G., \& Kehoe, E. J. (2003). Savings in classical conditioning in the rabbit as a function of extended extinction. Learning \& Behavior, 31, 49-68.

ZAKAY, D. (1990). The evasive art of subjective time measurement: Some methodological dilemmas. In R. A. Block (Ed.), Cognitive models of psychological time (pp. 59-84). Hillsdale, NJ: Erlbaum. 


\section{APPENDIX}

Secondary Task Analysis

Post hoc analyses were conducted to examine the spoken responses in the secondary task as a function of block of training. Only training trials in Experiment 2 were considered for those participants in the difficult training condition. The oral responses for 4 of the participants could not be scored, because of the quality of the recording. For the remaining 20 participants, two measures of performance were examined: accuracy and rate. For accuracy, a pair of successive letters spoken was scored as an error if the second letter was not three letters lower in the alphabet than the first letter, and the proportion of errors was computed across all pairs. For rate, the number of letters said per second was computed by counting the total number of letters spoken during a trial (with no consideration of accuracy) and dividing that total by the time in seconds that had elapsed during that trial (i.e., the time in seconds of the produced interval). The main effect of block was not significant for accuracy $\left[F(5,95)=2.18, p=.063 ; \eta^{2}=.10\right]$, nor was there a significant linear trend $[t(19)=-2.07, p=.053]$, but both results were close to significance. Specifically, the proportion of errors decreased monotonically across blocks (Block 1, .336; Block 2, .295; Block 3, .284; Block 4, .268; Block 5, .253; Block 6, .239). Likewise, the main effect of block was not significant for rate $\left[F(5,95)=1.78, p=.124 ; \eta^{2}=.09\right]$, nor was the linear trend $[t(19)=1.65, p=.116]$, but the number of letters said per second generally increased across blocks (Block 1 , .178; Block 2, .171; Block 3, .182; Block 4, .191; Block 5, .204; Block 6, .200). In any event, because performance on the secondary task at least showed a tendency toward improvement across blocks by both of these measures, there was no trade-off between performance on the secondary task and accuracy on the primary task (proportional absolute error on duration production), which showed significant improvement across blocks. Likewise, when accuracy on the primary task (proportional absolute error) and accuracy on the secondary task (the proportion of errors) were compared across participants, a small positive, but nonsignificant, correlation was found $[r(18)=.102]$. When the proportional absolute error on the primary task and the number of letters said per second in the secondary task were compared across participants, a larger, but still nonsignificant, positive correlation was found $[r(18)=.337]$, suggesting that those participants who went quickly through the secondary task tended to lose track of time, so that the intervals they produced for the primary task were less accurate.

(Manuscript received March 3, 2004;

revision accepted for publication November 30, 2004.) 